\title{
Prediction of cardiac function after weaning from ventricular assist devices
}

\author{
Hong Liang, MD, ${ }^{a}$ Hansheng Lin, MD, ${ }^{\mathrm{b}}$ Yuguo Weng, MD, ${ }^{\mathrm{c}}$ Michael Dandel, MD, ${ }^{\mathrm{c}}$ and Roland Hetzer, MD, $\mathrm{PhD}^{\mathrm{c}}$
}

Objective: We analyzed the factors influencing the cardiac function of patients after weaning from ventricular assist devices and established a prognostic index.

Method: A multivariate analysis was performed using the Cox proportional hazards model of the clinical parameters of 28 patients with end-stage heart failure before implantation of a ventricular assist device and directly before weaning from the device.

Results: After weaning from the ventricular assist devices, 14 of the 28 investigated patients showed stable cardiac function, and 12 had recurrent heart failure, but only 1 of them died before transplantation or implantation of another ventricular assist device, and 2 patients died of causes unrelated to heart failure. In addition to left ventricular ejection fraction, the duration of symptomatic heart failure and left ventricular intracavitary dimensions in diastole measured before ventricular assist devices weaning were the major factors influencing cardiac function after weaning. In the group of patients with heart failure duration less than 3 years, only 2 of 13 cases were recurrent, but in the group with heart failure duration more than 3 years, 10 of 15 cases were recurrent. In 10 patients with 40 to $50 \mathrm{~mm}$ left ventricular intracavitary dimensions in diastole before weaning, only 1 case was recurrent. In 10 patients with 51 to $55 \mathrm{~mm}$ left ventricular intracavitary dimensions in diastole, 3 cases were recurrent. In the group with left ventricular intracavitary dimensions in diastole more than $56 \mathrm{~mm}$, all 8 cases were recurrent. A prognostic index was calculated using the following formula: prognostic index $=-10.10+0.208$ (heart failure duration in years) +0.173 (pre-explantation left ventricular intracavitary dimensions in diastole in millimeters). In the group with a prognostic index less than 0 , only 2 of 16 cases were recurrent, and the rate of stable cardiac function in the 51 st month after weaning was $83.6 \%$. In the group with a prognostic index greater than 0,10 of 12 cases were recurrent, and the rate of stable function in the 57 th month was $0.0 \%$.

From the Department of Cardiothoracic Surgery, ${ }^{a}$ First Affiliated Hospital of Jinan University, Guangzhou, China; the Department of Medical Statistics, ${ }^{\mathrm{b}}$ Medical College of Jinan University, Guangzhou, China; and the Department of Cardiothoracic and Vascular Surgery, ${ }^{\mathrm{c}}$ Deutsches Herzzentrum Berlin, Berlin, Germany.

Received for publication April 15, 2005; revisions received July 30 , 2005; accepted for publication Aug 8, 2005.

Address for reprints: Hong Liang, MD, Department of Cardiothoracic Surgery, First Affiliated Hospital of Jinan University, 510630 Guangzhou, P.R. China (E-mail: lianghong58@hotmail.com).

J Thorac Cardiovasc Surg 2005;130:1555-60

$0022-5223 / \$ 30.00$

Copyright $(9) 2005$ by The American Association for Thoracic Surgery

doi:10.1016/j.jtcvs.2005.08.020

Conclusion: In patients with off-pump left ventricular ejection fraction greater than $40 \%$, the duration of symptomatic heart failure and the left ventricular intracavitary dimensions in diastole measured before weaning yield a useful index to predict long-term cardiac function after weaning from ventricular assist devices.

$\mathrm{T}$ he use of mechanical cardiac assist devices as a bridge to cardiac transplantation has been broadly reported, ${ }^{1-4}$ and their beneficial influence on the cardiac function of patients with end-stage heart failure has been established..$^{5-11}$ In March 1995 at the Deutsches Herzzentrum Berlin (German Heart Institute Berlin), a patient supported with a left ventricular assist device (LVAD) for 160 days showed significant improvement in cardiac function, and the LVAD was explanted. Cardiac function remained stable after LVAD explantation, and today the patient is doing well; this was the first case of its kind worldwide. In recent years, the number of reports of weaning from LVAD support after cardiac recovery has 


$\begin{array}{ll}\text { Abbreviations and Acronyms } \\ \beta_{1} \text {-adrenoreceptor } & =\mathrm{A}-\beta_{1} \text {-AAB } \\ \text { DCM } & =\text { dilated cardiomyopathy } \\ \text { ICM } & =\text { ischemic cardiomyopathy } \\ \text { LVAD } & =\text { left ventricular assist device } \\ \text { LVEF } & =\text { left ventricular ejection fraction } \\ \text { LVIDd } & =\text { left ventricular intracavitary } \\ & \text { dimensions in diastole } \\ \text { LVIDs } & =\text { left ventricular intracavitary } \\ & \text { dimensions in systole } \\ \text { PI } & =\text { prognostic index } \\ \text { VAD } & =\text { ventricular assist device }\end{array}$

increased, ${ }^{8,9,12-16}$ but there is little information about the cardiac function of the patients several years after weaning. This study analyzed the clinical data of 28 patients weaned from mechanical cardiac assist devices and assessed the influence of pre-explantation clinical parameters on the status of cardiac function after weaning. The aim was to find clinical parameters that can be used to predict stable recovery of cardiac function.

\section{Patients and Methods Patient Data}

Patient data were collected at the Deutsches Herzzentrum Berlin between March 1995 and January 2003. In this period, 32 patients were weaned from ventricular assist devices (VADs) after recovery of their cardiac function. There were 30 men and 2 women. Thirty patients had idiopathic dilated cardiomyopathy (DCM), without any angiographic evidence of coronary stenoses. Myocardial tissue specimens obtained in these 30 patients before LVAD implantation also showed no histologic signs of acute myocarditis, and virologic examinations did not reveal evidence of a virus infection that could have caused the myocardial disease. Polymerase chain reactions for enterovirus, adenoviruses, and cytomegalovirus were negative. In situ hybridization for the same viruses, and for coxsackievirus, Epstein-Barr virus, and herpes viruses, was negative in these patients. Only 1 of these 30 patients had a family history of DCM. The 31st weaned patient had angiographically proven ischemic cardiomyopathy (ICM), and the 32nd patient had myocarditis 6 months before LVAD implantation. The patients all had end-stage heart failure, and their symptoms could not be controlled by medication. Twenty-six patients received a Novacor 100 LVAD (Baxter Co, Oakland, Calif), 3 patients received a TCI LVAD (TCI HeartMate, Woburn, Mass), and 3 patients received a Berlin Heart LVAD, a Berlin Heart INCOR I axial flow pump (Berlin Heart AG, Berlin Germany), and a biventricular assist device, respectively. The ventricular assist period ranged from 22 to 794 days (mean, $150 \pm 150$ days).

During mechanical unloading all patients were treated with beta-blockers (metoprolol or carvedilol), angiotensin-converting enzyme inhibitors, aldosterone antagonists (spironolactone), lowdose loop diuretics (furosemide or torsemide), and digitalis. This treatment was also continued in all weaned patients.
At the end of the study, 16 patients had stable cardiac performance without any signs of deterioration of echocardiographic parameters (termed "stable" in the study). Twelve patients had a recurrence of heart failure (termed "recurrence" in the study) 2 to 57 months (mean, $15.8 \pm 19.3$ months) after pump explantation (10 underwent cardiac transplantation, 1 is on the waiting list after the implantation of another mechanical circulatory support system, and 1 died of recurrence of heart failure). In addition, 4 patients died of causes unrelated to heart failure (termed "death" in the study).

Indications for LVAD implantation were as follows: end-stage heart disease in patients on the waiting list for cardiac transplantation, deterioration of cardiac function, cardiogenic shock despite maximal medical therapy including inotropes and intra-aortic balloon pump intervention, and evidence of an early stage of multiple organ failure with right ventricular function still in a compensatory period.

Indications for device explantation were as follows: left ventricular ejection fraction (LVEF) increase more than $40 \%$ to $45 \%$; left ventricular intracavitary dimensions in diastole (LVIDd) decrease to less than $55 \mathrm{~mm}$; improvement of heart function parameters; approximately normal cardiothoracic ratio; and autoantibodies directed against the $\beta_{1}$-adrenoreceptor ( $\mathrm{A}-\beta_{1}$-AABs) no longer detectable in the LVAD recipient's serum. ${ }^{17}$ The effects of unloading on ventricular size, shape, and contraction were evaluated by weekly transthoracic echocardiographic examinations. If with the regularly running pump, without any inotropic drug therapy, the wall motion and diameters of the supported ventricle showed a tendency to normalization, the pulsatile pumps were set at the lowest pumping frequency and intermittently stopped for a few minutes ("off-pump" trial) to evaluate the heart without mechanical support. To prevent thrombus formation inside the pump, heparin was administrated intravenously before each off-pump trial. In addition, during the off-pump period the device was allowed to pump once per minute. In patients with axial flow pumps, we reduced the rotor speed to a value that results in a zero net flow over the time of 1 cardiac cycle. In patients with evidence of relevant cardiac recovery, such off-pump examinations (or trials with reduced rotor speed in the case of axial pumps) were conducted once per week until the final decision for VAD explantation was made. Between the off-pump trials the assist pumping rate was reduced step by step to load the LV gradually. At first the pumping frequency was reduced by 10 times per minute. If heart size, LVEF, and left ventricular wall thickness remained stable or normalized, and the off-pump trial did not reveal relevant alterations in ventricular size, the pumping rate could be reduced by a further 10 times per minute 1 week later until the lowest possible pumping rate (50 times/min) was reached. After this, if parameters of cardiac size and function measured by echocardiography during repeated off-pump trials in patients with pulsatile pumps or maximal reduction of rotor speed in those with axial pumps still remained nearly normalized, the explantation procedure could be performed. If left ventricular function could not be improved but deteriorated during this process, it was necessary to return to the original LVAD pumping rate. Off-pump echocardiographic data obtained without any inotropic drug therapy were decisive for the weaning procedure and LVEF off-pump values of greater than $40 \%$ were considered insufficient for reliable LVAD explantation. 
TABLE 1. Univariate analysis by Cox proportional hazards model

\begin{tabular}{lcccc}
\hline Prognostic factors & B & $\boldsymbol{P}$ & RR & 95\% CI for RR \\
\hline Age (y) & 0.064 & .040 & 1.067 & $1.003 \sim 1.134$ \\
HF duration (y) & 0.261 & .000 & 1.298 & $1.123 \sim 1.500$ \\
$\begin{array}{l}\text { Assist time (d) } \\
\begin{array}{l}\text { Pre-explantation } \\
\quad \text { LVEF (\%) }\end{array}\end{array}$ & 0.004 & .025 & 1.004 & $1.000 \sim 1.007$ \\
$\begin{array}{c}\text { Pre-explantation } \\
\quad \text { LVIDd (mm) }\end{array}$ & 0.112 & .013 & 0.894 & $0.819 \sim 0.977$ \\
$\begin{array}{c}\text { Pre-explantation } \\
\quad \text { LVIDs (mm) }\end{array}$ & 0.228 & .001 & 1.256 & $1.102 \sim 1.431$ \\
\hline
\end{tabular}

$B$, Regression coefficient; $R R$, $\exp (\mathrm{B})$, relative risk; $\mathrm{Cl}$, confidence interval; $H F$, heart failure; $L V E F$, left ventricular ejection fraction; $L V I D d$, left ventricular intracavitary dimensions in diastole; LVIDs, left ventricular intracavitary dimensions in systole.

To avoid excessive stimulation of the unloaded myocardium during the process of recovery, dobutamine stress echocardiography was not performed during the off-pump trials.

\section{Clinical Parameters}

The parameters investigated in the study for their potential prognostic value included the following: the patient's age, duration of heart failure, ventricular assist duration, heart rate, echocardiographic parameters (LVEF, LVIDd, left ventricular intracavitary dimensions in systole [LVIDs], and wall thickness), and hemodynamic parameters (cardiac index, pulmonary arterial pressure, and systemic blood pressure), all measured at the time of VAD implantation and before explantation at optimal recovery. Duration of heart failure was defined as the time period from the first appearance of clinically proved signs and symptoms of heart failure that necessitated medical treatment up to the date of VAD implantation. Periods of arrhythmia or electrocardiographic changes before the first evidence of congestive heart failure were not included in the calculation of heart failure duration.

\section{Inclusion and Exclusion Criteria}

Patients observed for more than 1 month after explantation and whose clinical data were complete were included in the study. There were 2 patients who died of causes unrelated to heart failure at 0.1 and 0.3 months after weaning. The first of these 2 patients had extensive infection at the LVAD conduit sites before weaning and died of septic shock; the second died of severe pulmonary bleeding. One other patient was observed for less than 1 month after explantation, and another patient's clinical data were not complete. These 4 patients were excluded from the study. Finally we studied 28 cases, comprising 14 patients with stable cardiac function, 12 patients with heart failure recurrence, and 2 patients who died of causes unrelated to heart failure (one died of pulmonary embolism and one died of left ventricular bleeding).

\section{Statistical Analysis}

Survival time was defined as the period from weaning to the end of follow-up. Statistical significance of the variables was first determined by univariate analysis using the Cox proportional
TABLE 2. Multivariate analysis by Cox proportional hazards model

\begin{tabular}{lcccc}
\hline Prognostic factors & B & $\boldsymbol{P}$ & $\mathbf{R R}$ & $\mathbf{9 5 \%}$ CI for $\mathbf{R R}$ \\
\hline $\begin{array}{l}\text { HF duration (y) } \\
\begin{array}{l}\text { Pre-explantation } \\
\text { LVIDd (mm) }\end{array}\end{array}$ & 0.208 & 0.012 & 1.231 & $1.046 \sim 1.450$ \\
\hline
\end{tabular}

$B$, Regression coefficient; $R R, \exp (\mathrm{B})$, relative risk; $\mathrm{Cl}$, confidence interval; $H F$, heart failure; $L V I D d$, left ventricular intracavitary dimensions in diastole.

hazards model. The variables that were statistically significant by univariate analysis were included in a multivariate analysis and confirmed using the Cox stepwise proportion hazards model. Survival time was estimated by the Kaplan-Meier method and was compared between groups divided in accordance with the different levels of variables that were statistically significant in multivariate analysis. The predictive model for prognosis was established according to the results of multivariate analysis. All analyses were performed using the SPSS 10.0 software (SPSS Inc, Chicago, Ill).

\section{Results}

\section{Univariate Analysis by Cox Proportional Hazards Model}

The prognosis was significantly influenced by 6 of the 13 variables submitted to univariate analysis (Table 1). The age, duration of heart failure, assist time, pre-explantation LVIDd, and pre-explantation LVIDs (LVIDd and LVIDs measured directly before explantation) were seen to be risk factors, because the risk of recurrences increased with higher level of the variables (relative risk $>1.0, P<.05$ ), whereas pre-explantation LVEF was predictive of absence of heart failure recurrence (relative risk $=0.894, P=.013$ ).

\section{Multivariate Analysis by Cox Proportional Hazards Model}

Multivariate analysis involving the 6 significant factors determined by univariate analysis identified 2 highly prognostic variables, which were duration of heart failure and pre-explantation LVIDd (Table 2). The frequency distribution of duration of heart failure was as follows: 9 cases shorter than 2 years, 4 cases ranging from 2 to 3 years, 7 cases ranging from 4 to 5 years, and 8 cases ranging from 6 to 13 years. The average duration of heart failure was $4.3 \pm$ 3.8 (mean $\pm \mathrm{SD}$ ) years and 4.0 (median) years.

\section{Survival Analysis}

Survival analysis was performed according to the variables of heart failure duration and pre-explantation LVIDd.

Two groups were distinguished by duration of heart failure: 3 years or less (0-3) and greater than 3 years (4-13). In the 3 years or less group $(n=13)$ there were 2 recurrences and 11 cases of stable cardiac function, whereas in the greater than 3 years group $(n=15)$ there were 10 recurrences, 3 cases of stable function, and 2 deaths. Stable function rates were ana- 


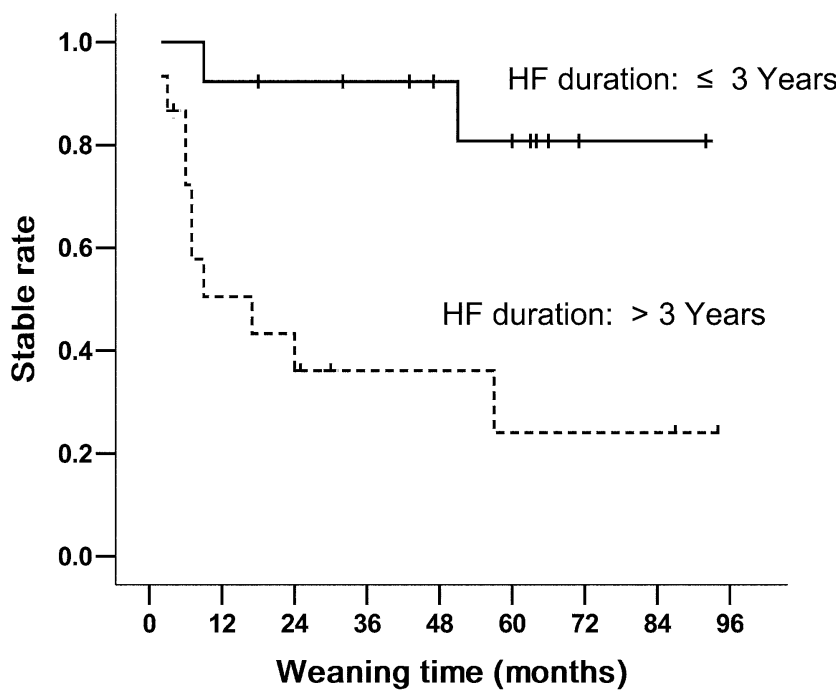

Figure 1. Heart failure duration and stable cardiac function rate. $H F$, Heart failure.

lyzed in each group: $92.3 \%$ for 9 months and $80.8 \%$ for 51 months in the 3 years or less group; $50.7 \%$ for 9 months, $36.1 \%$ for 24 months, and $24.1 \%$ for 57 months in the greater than 3 years group (Figure 1).

Three groups were distinguished by pre-explantation LVIDd with 1 recurrence, 8 "stable," and 1 death in 10 cases in the group with less than $50 \mathrm{~mm}(40-50 \mathrm{~mm}) ; 3$ recurrences, 6 "stable," and 1 death in 10 cases in the group with 51 to $55 \mathrm{~mm}$; recurrence in all 8 cases in the group with 56 to $66 \mathrm{~mm}$ (Figure 2).

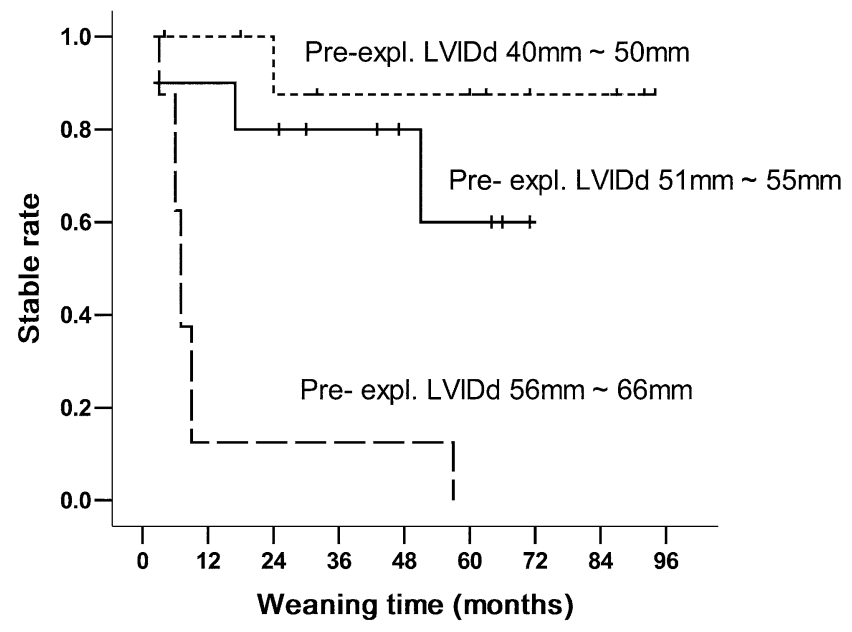

Figure 2. LVIDd measured before explantation (pre-expl. LVIDd) and rate of stable cardiac function. LVIDd, Left ventricular intracavitary dimensions in diastole.

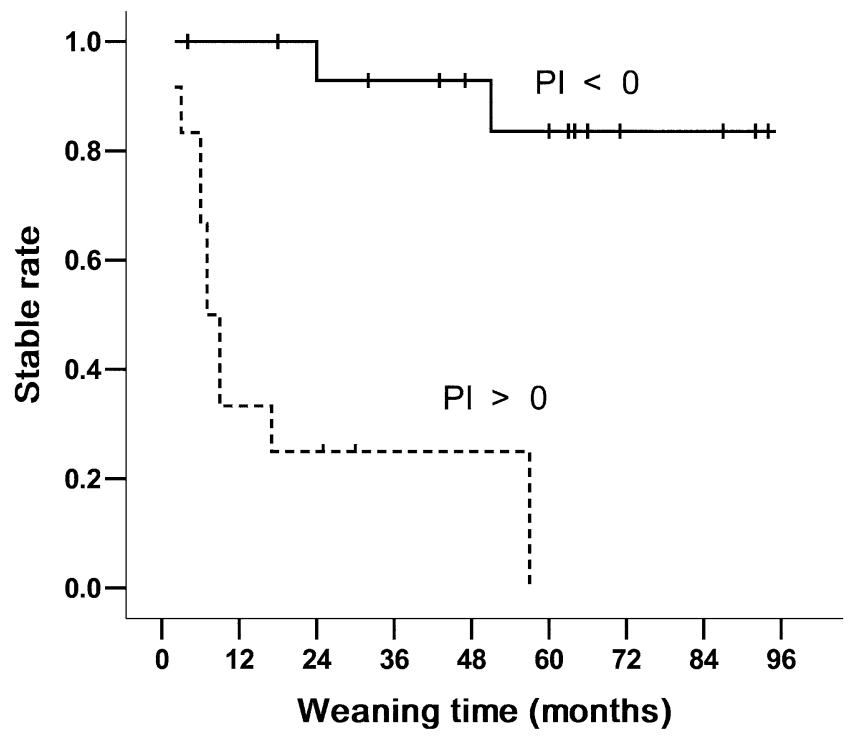

Figure 3. PI and stable cardiac function rate. PI, Prognosis index.

\section{Predictive Model for Prognosis}

The model was established according to the results of multivariate analysis involving the 2 variables of heart failure duration and pre-explantation LVIDd. The value of the prognostic index (PI) for predicting the survival of individuals was calculated as follows: ${ }^{18}$

$$
\begin{gathered}
P I=\beta_{0}+\beta_{1} X_{1}+\beta_{2} X_{2}+\cdots+\beta_{m} X_{m} \\
\beta_{0}=-\left(\beta_{1} \bar{X}_{1}+\beta_{2} \bar{X}_{2}+\cdots+\beta_{m} \bar{X}_{m}\right)
\end{gathered}
$$

$\beta_{0}$ is the constant for all the patients, and $\beta$ and $\mathrm{X}$ present the regression coefficients and variables. The model in this study was as follows:

$$
\begin{gathered}
\beta_{1}=0.208, \bar{X}_{1}=4.286, \beta_{2}=0.173, \bar{X}_{2}=53.250 \\
\beta_{0}=-(0.208 \times 4.286+0.173 \times 53.250)=-10.10
\end{gathered}
$$

$\mathrm{PI}=-10.10+0.208$ (heart failure duration in years) + 0.173 (pre-explantation LVIDd in millimeters).

Two groups were distinguished by the PI: one with PI below 0 and the other with a PI above 0 . In the group with PI of less than 0 there were 2 recurrences of 16 cases, and the "stable" rate was $92.9 \%$ for 24 months and $83.6 \%$ for 51 months. In the group with a PI of more than 0 there was recurrence in 10 of 12 patients, and the "stable" rate was $66.7 \%$ for 6 months, $33.3 \%$ for 9 months, $25.0 \%$ for 17 months, and $0.0 \%$ for 57 months (Figure 3).

\section{Discussion}

The indication for VAD implantation was bridging to cardiac transplantation. ${ }^{8}$ Up to now no attempts have been 
made to use VADs electively with the aim of myocardial recovery only, and no study has established which patients in whom end-stage heart disease may achieve myocardial recovery with VADs. Both in previous reports and in this study the majority of cases of myocardial recovery with LVAD were seen in patients with DCM. During the past 9 years, $24 \%$ of our patients with DCM supported by VADs were weaned from mechanical support. At 3 years after VAD explantation, freedom from recurrent heart failure was found in only $70 \%$ of the weaned patients, so that finally only $13 \%$ of patients with end-stage DCM showed longterm recovery of cardiac function.

The echocardiographic data were decisive for the weaning procedure. The disappearance of A- $\beta_{1}-\mathrm{AABs}$ was helpful to optimize the follow-up to select the optimal time for explantation, after myocardial improvement had reached its maximum. Of the weaned patients, $96.4 \%$ tested positive for $\mathrm{A}-\beta_{1}-\mathrm{AABs}$ before VAD implantation, and in $96.3 \%$ of the patients who tested positive, A- $\beta_{1}$-AABs disappeared after 3 to 31 weeks of ventricular unloading. However, between patients with and without heart failure recurrence during the first 3 years after weaning there were no significant differences for serum levels of A- $\beta_{1}$-AABs at the time of LVAD implantation or for the disappearance time of A- $\beta_{1}$-AABs during VAD support.

Implantation of an LVAD is initially almost always as a bridge to transplantation. The VAD can be explanted only if cardiac function parameters improve during the assist period and it is considered on the basis of comprehensive clinical assessment and examination that the patient's own heart can again tolerate loading and pump blood to the organs. If cardiac function remains normalized after explantation, the VAD can be considered retrospectively as a bridge to myocardial recovery.

In the wake of increasing application of VADs, the reports of improvement and recovery of severely impaired myocardium during unloading by mechanical cardiac support enabling weaning from VAD are gradually increasing. In the follow-up, some of the patients weaned from VAD required another period of mechanical support or underwent heart transplantation because of recurrence of heart failure. The possibility of predicting cardiac function status after explantation would be extremely valuable, but up until now this has scarcely been studied because of the small number of cases. The data collected during this study represent the largest group of weaned patients in the world from one center. The study made a retrospective analysis of clinical parameters to find an index with prognostic value for cardiac function after weaning. The results can provide reference values for clinical work.

The study suggests that both the duration of heart failure and LVIDd measured before VAD weaning have a clear influence on the stability of cardiac function after weaning.
The result is easy to explain clinically. The patients with long duration of heart failure ( $>3$ years) usually have severely impaired myocardium. Serious changes caused by ventricular remodeling, such as myocardial hypertrophy, denaturalization, and fibrosis and collagen deposit in the intracellular matrix, become more serious with prolongation of the clinical course and repeated episodes of heart failure. Although the VAD can reverse adverse ventricular remodeling, the long clinical course makes this process difficult, and if the reversal is incomplete, it eventually causes the recurrence of heart failure after VAD explantation. The small amount of histologic data obtained from our weaned patients was insufficient to evaluate their predictive value for functional cardiac recovery. However, in 10 patients we evaluated by histomorphometry the amount of fibrosis in both myocardial samples obtained during LVAD implantation and biopsy material taken before pump explantation; in only 1 of these patients was there no reduction of fibrosis during unloading. Also, regression of myocardial hypertrophy during unloading was shown in 9 of these 10 patients.

Explantation of the VAD could be considered only when LVIDd reached less than $50 \mathrm{~mm}$, but in the process of weaning, the data may fluctuate. In the study it was shown that if LVIDd at explantation time was less than $50 \mathrm{~mm}$, the prognosis would be good; if it was more than $55 \mathrm{~mm}$, the outcome was worse even if the LVEF was greater than $40 \%$. This means that the advantageous influence of the VAD on heart function is reflected also by this echocardiographic parameter. The more LVIDd approaches the normal range during circulatory support, the greater the chances are that the myocardium may completely recover. These results provide scientific data for the selection of suitable weaning cases in future clinical work, thus raising the lasting stability rate of heart function after VAD explantation.

According to our PI, in patients with duration of heart failure longer than 3 years before VAD implantation, VAD explantation after functional recovery will be risky as long as the LVIDd is $55 \mathrm{~mm}$ or greater. Although in the past we did not include the duration of heart failure in our weaning criteria, our present decision making in patients with signs of relevant cardiac improvement during ventricular mechanical unloading is markedly influenced by the history of heart failure. Therefore, in addition to the ventricular ejection fraction, the PI is also used to evaluate the potential weaning success.

Although the recurrence of heart failure after VAD explantation can be treated by implanting another VAD or by heart transplantation, this not only increases the costs of medical care but also involves the operative risk of cardiac transplantation. The predictive model established in this study was obtained by considering the clinical duration of heart failure and LVIDd measured before VAD explantation. The model not only assessed the persistence of stable 
heart function after explantation but also provided a reference framework for the surgeon in selecting device explantation or continuing to wait for transplantation. Myocardial biopsy might theoretically be more significant for assessing prognosis, but the process would carry a large risk for patients, especially at the time of explantation, and for this reason it is rarely considered for such cases. The parameters investigated by our study are easily obtained, and the index will provide valuable reference data for clinical decision making.

Our data show that there are reliable criteria that indicate complete and stable cardiac recovery on VADs and also reliably predict the long-term success of weaning from a VAD primarily designated as "bridge to transplantation." Unfortunately, according to our data it is still not possible to prospectively identify those patients who would benefit from elective VAD insertion primarily designed as a "bridge to myocardial recovery."

Because all patients with implanted VADs received practically the same medical therapy during ventricular unloading, no conclusions can be drawn on the contribution of medication to the process of myocardial recovery.

We thank Dr Johannes Mueller for his assistance in this study, Ms Christiane Schulz for clinical data collection, and Ms Anne M. Gale for editorial assistance.

\section{References}

1. Hetzer R, Hennig E, Schiessler A, Friedel N, Warnecke H, Adt M. Mechanical circulatory support and heart transplantation. J Heart Lung Transplant. 1992;11:S175-81.

2. Korfer R, el-Banayosy A, Posival H, Minami K, Korner MM, Arusoglu L, et al. Mechanical circulatory support the Bad Oeynhausen experience. Ann Thorac Surg. 1995;59:S56-63.

3. Hunt S, Frazier OH, Myers TJ. Mechanical circulatory support and cardiac transplantation. Circulation. 1998;97:2079-90.

4. Goldstein DJ, Oz MC, Rose EA. Implantable left ventricular assist devices. N Engl J Med. 1998;339:1522-33.
5. Liven HR, Oz MC, Catanese KA, Rose EA, Burkhoff D. Transient normalization of systolic and diastolic function after support with a left ventricular assist device in a patient with dilated cardiomyopathy. J Heart Lung Transplant. 1996;15:840-2.

6. Rose EA, Frazier OH. Resurrection after mechanical circulatory support [editorial]. Circulation. 1997;96:393-5.

7. Nakatani S, McCarthy PM, Kottke-Marchant K, Harasaki H, James $\mathrm{KB}$, Savage RM, et al. Left ventricular echocardiographic and histologic changes: impact of chronic unloading by an implantable ventricular assist device. J Am Coll Cardiol. 1996;27:894-901.

8. Hetzer R, Mueller J, Weng YG, Wallukat G, Spiegelsberger S, Loebe M. Cardiac recovery in dilated cardiomyopathy by unloading with a left ventricular assist device. Ann Thorac Surg. 1999;68:742-9.

9. Kumpati GS, McCarthy PM, Hoercher KJ. Left ventricular assist device as a bridge to recovery: present status. J Card Surg. 2001;16: 294-301.

10. Dohmen PM, Laube HR, de Jonge KC, Konnertz W. A reliable test to detect impending pump failure, during long-term support on the Novacor ${ }^{\circledR}$ N100 left ventricular assist system. Tex Heart Inst J. 2001; 28:139-41.

11. Morales DLS, Catanese KA, Helman DN, Williams MR, Weinberg A, Goldstein DJ, et al. Six-year experience of caring for forty-four patients with a left ventricular assist device at home: safe, economical, necessary. J Thorac Cardiovasc Surg. 2000;119:251-9.

12. Mueller J, Wallukat G, Weng YG, Dandel M, Spiegelsberger S, Semrau $\mathrm{S}$, et al. Weaning from mechanical cardiac support in patients with idiopathic dilated cardiomyopathy. Circulation. 1997;96:542-9.

13. Farrar DJ, Holman WR, McBride LR, Kormos RL, Icenogle TB, Hendry PJ, et al. Long-term follow-up of Thoratec ventricular assist device bridge-to-recovery patients successfully removed from support after recovery of ventricular function. J Heart Lung Transplant. 2002; 21:516-21.

14. Davies JE, Kirklin JK, Pearce FB, Rayburn BK, Winokur TS, Holman WL. Mechanical circulatory support for myocarditis: how much recovery should occur before device removal? J Heart Lung Transplant. 2002;21:1246-9.

15. Frazier $\mathrm{OH}$, Myers TJ. Left ventricular assist system as a bridge to myocardial recovery. Ann Thorac Surg. 1999;68:734-41.

16. Hetzer R. Mueller J, Weng YG, Meyer R, Dandel M. Bridging-torecovery. Ann Thorac Surg. 2001;71:S109-13.

17. Mueller J, Hetzer R. Left ventricular recovery during left ventricular assist device support: In: Goldstein DJ, Oz MC, editors. Cardiac assist devices. Armonk (NY): Futura Publishing Co; 2000. p. 121-35.

18. Chen GM. Survive analysis: In: Sun GQ, Xu YY, editors. Medical statistics. Peking, China: People's Health Publishing Co; 2002. p. 272-94. 